\title{
Diagnostic Quality of 3D T2-SPACE Compared with T2-FSE in the Evaluation of Cervical Spine MRI Anatomy
}

\author{
(D)F.H. Chokshi, (D) G. Sadigh, (D)W. Carpenter, and (D).W. Allen
}

\begin{abstract}
BACKGROUND AND PURPOSE: Spinal anatomy has been variably investigated using 3D MRI. We aimed to compare the diagnostic quality of T2 sampling perfection with application-optimized contrasts by using flip angle evolution (SPACE) with T2-FSE sequences for visualization of cervical spine anatomy. We predicted that T2-SPACE will be equivalent or superior to T2-FSE for visibility of anatomic structures.
\end{abstract}

MATERIALS AND METHODS: Adult patients undergoing cervical spine MR imaging with both T2-SPACE and T2-FSE sequences for radiculopathy or myelopathy between September 2014 and February 2015 were included. Two blinded subspecialty-trained radiologists independently assessed the visibility of 12 anatomic structures by using a 5-point scale and assessed CSF pulsation artifact by using a 4-point scale. Sagittal images and 6 axial levels from C2-T1 on T2-FSE were reviewed; 2 weeks later and after randomization, T2-SPACE was evaluated. Diagnostic quality for each structure and CSF pulsation artifact visibility on both sequences were compared by using a paired $t$ test. Interobserver agreement was calculated $(\kappa)$.

RESULTS: Forty-five patients were included (mean age, 57 years; 40\% male). The average visibility scores for intervertebral disc signal, neural foramina, ligamentum flavum, ventral rootlets, and dorsal rootlets were higher for T2-SPACE compared with T2-FSE for both reviewers $(P<.001)$. Average scores for remaining structures were either not statistically different or the superiority of one sequence was discordant between reviewers. T2-SPACE showed less degree of CSF flow artifact $(P<.001)$. Interobserver variability ranged between $-0.02-0.20$ for T2-SPACE and $-0.02-0.30$ for T2-FSE (slight to fair agreement).

CONCLUSIONS: T2-SPACE may be equivalent or superior to T2-FSE for the evaluation of cervical spine anatomic structures, and T2SPACE shows a lower degree of CSF pulsation artifact.

ABBREVIATIONS: C-spine = cervical spine; SPACE = sampling perfection with application-optimized contrasts by using flip angle evolution

$\mathrm{H}$ istorically, cervical spine (C-spine) MR imaging has included 2D T1WI and T2WI sequences that allow single-plane visualization of soft tissue and osseous structures. The advent of 3D MR imaging sequences has allowed MPR visualization after sin-

Received September 8, 2016; accepted after revision November 23.

From the Department of Radiology and Imaging Sciences, Division of Neuroradiology (F.H.C., G.S., J.W.A.), Department of Biomedical Informatics (F.H.C.), Department of Radiology and Imaging Sciences, Division of Musculoskeletal Radiology (W.C.), and Department of Neurology (J.W.A.), Emory University School of Medicine, Atlanta, Georgia.

This work is supported, in part, by the Association of University Radiologists (AUR) General Electric Academic Radiology Research Fellowship (GERRAF) grant. Dr. Chokshi is an AUR GERRAF Fellow from 2015 to 2017.

Paper previously presented at: Annual Meeting of the American Society of Neuroradiology and the Foundation of the ASNR Symposium, May 21-26, 2016; Washington, DC.

Please address correspondence to Falgun H. Chokshi, MD, MS, Department of Radiology and Imaging Sciences, Division of Neuroradiology, Emory University School of Medicine, 1364 Clifton Rd NE, Atlanta, GA 30322; e-mail: falgun.chokshi@ emory.edu; @FalgunChokshiMD

http://dx.doi.org/10.3174/ajnr.A5080 gle-plane acquisition. Although 3D sequences have historically been based on GRE techniques, the advent of FSE 3D sequences, such as sampling perfection with application-optimized contrasts by using flip angle evolution (SPACE sequence; Siemens, Erlangen, Germany), affords a potential new means of evaluating anatomy and pathology. The SPACE sequence is a proprietary 3D FSE sequence and is analogous to the VISTA (volume isotropic turbo spin-echo acquisition [Philips Healthcare, Best, the Netherlands]) sequence or Cube sequence (GE Healthcare, Milwaukee, Wisconsin). ${ }^{1}$

Studies have evaluated the advantages of 3D T2WI sequences in brain ${ }^{2}$ and head and neck imaging ${ }^{3,4}$; however, there have only been a small number of studies investigating their value in spine MR imaging. ${ }^{5-7}$ Most of these studies have compared the visualization of degenerative disease of the lumbar spine on conventional T2-FSE sequences versus 3D T2WI sequences. Lee et $\mathrm{al}^{7}$ reported no statistically significant difference between the sensitivities of T2-FSE and 3D T2-SPACE for the detection of neural 
foraminal stenosis, spinal canal stenosis, and nerve compression at 3T. Furthermore, Blizzard et $\mathrm{al}^{5}$ found a high degree of interobserver agreement between T2-FSE and T2-SPACE images for the evaluation of spinal canal stenosis, disc herniation, and degenerative changes. A similar study comparing T2-FSE and T2SPACE also found high interobserver agreement for the visualization of C-spine degenerative disease. ${ }^{6}$

To our knowledge, Meindl et $\mathrm{al}^{8}$ published the only study comparing the visualization of C-spine normal anatomic structures and CSF visualization on T2-FSE and T2-SPACE in 15 healthy volunteers (mean age, 28.4 years) and found statistically significant better visibility of CSF, intraspinal nerve roots, and neural foraminal structures on T2-SPACE. To date, however, there has been no similar study to evaluate anatomic visualization in clinically symptomatic patients. Such a study would help determine the replicability of the previous findings and generate further hypotheses about and support for how T2-SPACE could be used and integrated into routine spine MR imaging while maintaining appropriate workflow. Moreover, we felt it is important to establish visibility of anatomy before focusing on pathology because the former is the foundation of the latter.

Therefore, we aimed to adapt the methods of Meindl et al ${ }^{8}$ and evaluated clinical C-spine MRIs to assess the visualization of Cspine anatomy in a clinical patient population. We predicted that T2-SPACE would be equivalent or superior to T2-FSE for the evaluation of C-spine anatomic structures and that T2-SPACE would show a lesser degree of CSF flow artifact.

\section{MATERIALS AND METHODS \\ Patient Selection}

This retrospective study was approved by our institutional review board, and a waiver of consent was granted. We searched our institutional radiology data base for all C-spine MRIs without contrast performed on a 1.5T Aera scanner (Siemens, Erlangen, Germany) at 1 of our 2 university hospitals between September 1, 2014, and February 28, 2015. We limited the search to this scanner because it has a diagnostic-quality T2-SPACE sequence that is routinely acquired as part of the clinical C-spine MR imaging protocol. We included consecutive patients aged 18 years or older who underwent $\mathrm{C}$-spine MR imaging without contrast with both T2-FSE and T2-SPACE sequences for the indication of radiculopathy, myelopathy, or neck pain, which was determined by searching the indication on the scan requisition as filled out by the ordering physician. Using information from the electronic health record, patients were excluded if they had any history of malignancy, C-spine infection, C-spine surgery, and/or surgical instrumentation. Fig 1 shows the patient selection characteristics. $\mathrm{Pa}-$ rameters for the T2-FSE and T2-SPACE sequences are listed in Table 1.

\section{Anatomic Visualization and Scoring}

Using a paired study design, 2 reviewers blinded to the clinical presentation and C-spine MR imaging results (but not the patient demographics displayed on the PACS) independently assessed the visibility of 12 anatomic structures (Fig 2) and CSF flow artifact on the sagittal view and 6 axial levels spanning C2-T1 on T2-FSE. To decrease recall bias, T2-SPACE was evaluated at least 2 weeks

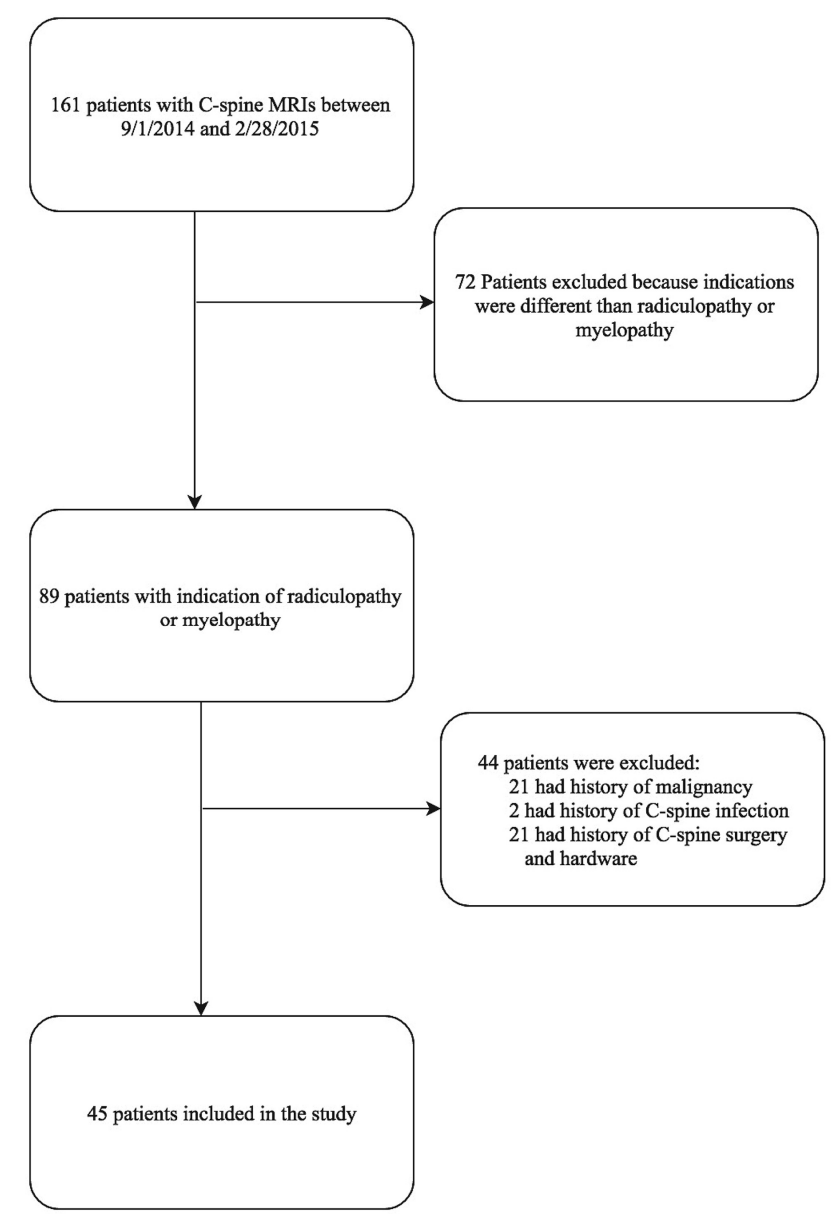

FIG 1. Patient selection flowchart.

Table 1: 1.5T MRI parameters for T2-FSE and T2-SPACE sequences

\begin{tabular}{lccc}
\hline \multicolumn{1}{c}{ Sequence } & T2-FSE & T2-FSE & T2-SPACE \\
\hline Orientation & Axial & Sagittal & Sagittal \\
TR (ms) & 3370 & 3140 & 1500 \\
TE $(\mathrm{ms})$ & 79 & 84 & 120 \\
FA (degrees) & 150 & 150 & Variable \\
FOV (mm) & 100 & 100 & 100 \\
Matrix & $256 \times 182$ & $384 \times 269$ & $256 \times 256$ \\
Voxel size (mm) & 3 & 3 & 1 \\
Sections & 40 & 15 & 80 \\
TA (min:sec) & $1: 44$ & $2: 46$ & $4: 00$ \\
\hline
\end{tabular}

Note:-TA indicates acquisition time; FA, flip angle.

later, and the subject order was randomized (paired study de$\left.\operatorname{sign}^{9}\right)$. Subsequently, each reviewer generated 8190 total visibility scores. Both reviewers were subspecialty-trained attending radiologists, each with more than 10 years of experience. Reviewer 1 was a musculoskeletal radiologist (W.C.) and reviewer 2 was a neuroradiologist (J.W.A.).

The C-spine anatomic structures were evaluated by using a 5 -point scale ( $0=$ not visible, $4=$ excellent visibility), and CSF flow artifact was evaluated by using a 4 -point scale $(0=$ severe artifact, $3=$ no artifact), both adapted from Meindl et al. ${ }^{8}$

\section{Statistical Analysis}

Differences between the visibility scores for the 2 sequences were tested for statistical significance by using a paired $t$ test for the scores documented for each of the 12 anatomic structures and the 
CSF flow artifact on sagittal view and 6 axial levels for each reviewer separately. Interobserver agreement of the 2 reviewers for each of the sequences (T2-FSE and T2-SPACE) was measured by using the Cohen $\kappa$ correlation coefficient. ${ }^{10}$ All statistical calculations were performed by using STATA/SE version 14.1 (StataCorp, College Station, Texas). $P<.05$ was considered statistically significant.

\section{RESULTS}

Forty-five patients met our inclusion criteria (Fig 1). The mean age was 57 years (SD, 15 years), ranging from 22-82 years, and $40 \%$ of the patients were men. As depicted in Table 2, average visibility scores for intervertebral disc signal, neural foramina, ligamentum flavum, ventral rootlets, and dorsal rootlets were higher for T2-SPACE compared with T2-FSE for both reviewers $(P<.001)$. For both reviewers, T2-SPACE showed significantly less CSF flow artifact compared with T2-FSE $(P<.001)$ (Fig 3).

Average scores for the remaining anatomic structures were either not statistically different or the superiority of one sequence was discordant between both reviewers. Reviewer 1 scored the bone marrow signal $(P<.001)$, anterior longitudinal ligament

\section{Osseous/Disc Structures \\ Bone Marrow Signal \\ Intervertebral Disc Signal \\ Facet Joints \\ Neural Foramina}

Ligamentous Structures

$A L L$

$P L L$

Ligamentum Flavum

Interspinous Ligament

\section{Cord, Nerves \\ Spinal Cord Signal \\ Ventral Rootlets \\ Dorsal Rootlets \\ Dorsal Root Ganglia}

FIG 2. C-spine anatomic structures evaluated by reviewers. ALL indicates anterior longitudinal ligament; PLL, posterior longitudinal ligament.

Table 2: Average visibility score for anatomic structures and CSF flow artifact comparing T2-FSE with T2-SPACE sequences

\begin{tabular}{|c|c|c|c|c|c|c|}
\hline & \multicolumn{3}{|c|}{$\begin{array}{c}\text { Reviewer } 1 \\
\text { (Musculoskeletal) }\end{array}$} & \multicolumn{3}{|c|}{$\begin{array}{c}\text { Reviewer } 2 \\
\text { (Neuroradiology) }\end{array}$} \\
\hline & T2-FSE & T2-SPACE & $P$ Value & T2-FSE & T2-SPACE & $P$ Value \\
\hline Bone marrow signal & 2.10 & 2.00 & $<.001$ & 2.94 & 2.96 & .34 \\
\hline Intervertebral disc & 2.00 & 2.50 & $<.001$ & 2.85 & 2.95 & $<.001$ \\
\hline Facet joints & 1.95 & 1.95 & .85 & 2.90 & 3.07 & $<.001$ \\
\hline Neural foramina & 1.90 & 1.97 & $<.001$ & 2.87 & 3.29 & $<.001$ \\
\hline Anterior longitudinal ligament & 0.23 & 0.16 & .004 & 2.83 & 2.92 & $<.001$ \\
\hline Posterior longitudinal ligament & 0.69 & 0.50 & $<.001$ & 2.91 & 3.00 & $<.001$ \\
\hline Ligamentum flavum & 1.41 & 1.78 & $<.001$ & 2.90 & 2.99 & $<.001$ \\
\hline Interspinous ligament & 1.15 & 1.36 & $<.001$ & 1.99 & 2.00 & .73 \\
\hline Spinal cord signal & 1.93 & 1.98 & .01 & 2.80 & 2.40 & $<.001$ \\
\hline Ventral rootlets & 0.39 & 1.43 & $<.001$ & 0.41 & 1.67 & $<.001$ \\
\hline Dorsal rootlets & 0.50 & 1.87 & $<.001$ & 0.55 & 2.35 & $<.001$ \\
\hline Dorsal root ganglia & 1.38 & 1.88 & $<.001$ & 1.11 & 2.64 & $<.001$ \\
\hline CSF flow artifact ${ }^{a}$ & 1.65 & 2.93 & $<.001$ & 1.69 & 2.8 & $<.001$ \\
\hline
\end{tabular}

a Please note, higher scores of CSF flow artifact correspond to less degree of artifact.
$(P=.004)$, and posterior longitudinal ligament higher for T2FSE, indicating better visualization compared with T2-SPACE. For this reviewer, there was no statistically significant difference in the visualization of the facet joints $(P=.85)$; conversely, reviewer 2 scored T2-SPACE higher than T2-FSE for facet joint visualization $(P<.001)$. Reviewer 2 scored the spinal cord signal $(P<.001)$ higher for T2-FSE compared with T2-SPACE, and there was no statistically significant difference in the visualization of the bone marrow signal $(P=.34)$ and the interspinous ligaments $(P=.73)$. Interobserver agreement ranged between $\kappa$ values of $-0.02-0.20$ for T2-SPACE and $-0.02-0.30$ for T2-FSE, consistent with slight to fair agreement for both sequences. ${ }^{10}$

\section{DISCUSSION}

In this study, we compared the visibility scores of 12 anatomic structures of the cervical spine and CSF pulsation artifact on T2FSE and T2-SPACE MR imaging sequences. Five anatomic structures, namely the intervertebral disc, neural foramina, ligamentum flavum, ventral rootlets, and dorsal rootlets, were better seen on T2-SPACE compared with T2-FSE for both reviewers, and CSF pulsation artifact was less on T2-SPACE. The remaining structures showed statistical equivalency in visualization or discordance in visualization between both reviewers.

This is the second study to focus on visualization of normal anatomic structures of the $\mathrm{C}$-spine at 1.5T MR imaging strength by using T2-SPACE. Compared with the first such study by Meindl et al, ${ }^{8}$ which included only healthy volunteers, ours included older patients with clinical indications warranting C-spine MR imaging and is arguably more clinically relevant. Similar to Meindl et al, ${ }^{8}$ we also found that intraspinal structures (rootlets) and neural foramina were better visualized with T2-SPACE, while also evaluating additional anatomic structures not evaluated in that study, namely the ligamentum flavum and longitudinal ligaments.

Comparable with Meindl et al, ${ }^{8}$ our study also found less CSF pulsation artifact (better CSF visibility) on T2-SPACE in comparison with T2-FSE. Conventional T2-FSE images are often wrought with pulsation artifact, ${ }^{11}$ which greatly diminishes their utility in visualizing thecal sac contents, especially small structures such as the rootlets. Many patients undergo invasive CT myelography ${ }^{12}$ or contrast-enhanced MR myelography ${ }^{13}$ to interrogate these structures. The ability of T2-SPACE to better identify the rootlets could be helpful in clinical diagnosis and surgical planning because no intrathecal contrast is needed and there is no radiation exposure. This "myelographic" application of T2-SPACE should be explored via prospective comparative trials with CT and MR myelography.

In contrast to studies of degenerative disease by using T2-SPACE ${ }^{2-7}$ and the MR imaging anatomy study by Meindl et $\mathrm{al}^{8}{ }^{8}$ our interobserver agreement for T2-SPACE ranged from slight to fair. It is unclear if this reflects any underlying difference in experience with this se- 


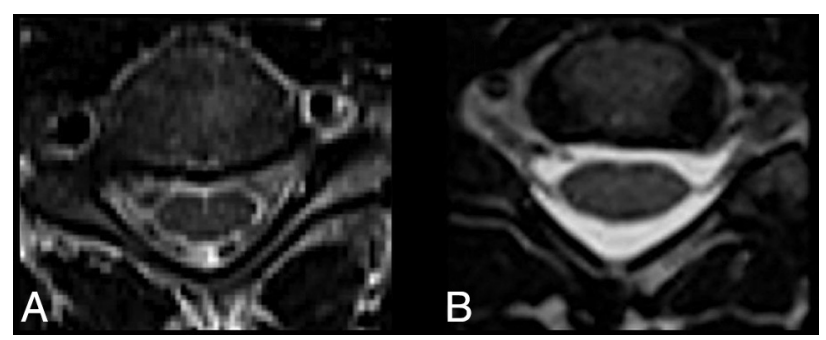

FIG 3. CSF visualization on T2-FSE and T2-SPACE. There is considerable CSF pulsation artifact on axial T2-FSE $(A)$ compared with no such artifact on axial T2-SPACE (B).

quence between the 2 reviewers. Reviewer 1 (attending musculoskeletal radiologist) had no experience with this newer sequence, whereas reviewer 2 (attending neuroradiologist) had approximately 1 year of experience. It is also unclear if this difference may reflect an underlying learning curve associated with the T2SPACE sequence or may be related to the different approaches to spine MR imaging interpretation between these 2 subspecialties. These questions could be assessed in future studies by testing multiple reader interobserver agreement after various amounts of experience with the sequence. In addition, future studies may benefit from training observers before the onset of the study to establish baselines for the measurements and thereby improve interobserver agreement.

Interestingly, we also found slight to fair interobserver agreement for the T2-FSE sequence. Although this finding has not been reported thus far for C-spine MRIs, a 2005 study by van Rijn et $\mathrm{al}^{14}$ found greater than $50 \%$ interobserver disagreement when they evaluated lumbar spine MRIs for disc herniation. That study included only neuroradiology-trained reviewers and examined degenerative lumbar spine disease (defined as osteophyte at endplates, disc herniation, central canal stenosis, and lateral recess stenosis). Interobserver studies of C-spine MR imaging have reported a wide range of agreement for detection of degenerative disease on T2-FSE or T2-SPACE. ${ }^{15-18}$ None, however, have reported interobserver agreement for T2-FSE visualization of Cspine anatomic structures. Our interobserver agreement results may represent the first description of visualization and detection differences of C-spine MR imaging anatomy on T2-FSE between musculoskeletal radiologists and neuroradiologists.

Our study limitations are as follows:

1) We used a retrospective study design, which raises the concern for selection bias and the presence of unknown confounders, both of which can be better addressed by using a prospective, randomized design;

2) Although we used a paired study design, we assessed visualization with only 2 reviewers. Future studies could use a multireader design, which would allow better evaluation of interobserver agreement and allow a lower sample size to find statistical significance ${ }^{19}$; and,

3) For T2-SPACE evaluation, we did not compare visualization scores between inexperienced versus experienced readers.

We feel the biggest advantage of T2-SPACE is the ability to acquire isotropic imaging data at millimeter or sub-millimeter section thickness with a single sagittal acquisition followed by multiplanar reformats. This is in contradistinction to conventional T2-FSE imaging, where the increased section thickness, and the variable addition of skip sections in some clinical settings, leads to an averaging of the area imaged rather than depicting the true anatomy. In addition, the artifacts that can cause diagnostic problems on T2-FSE tend to stem from CSF pulsation, which is minimized on T2-SPACE.

Potential roles for the T2-SPACE sequence may include, but are not limited to: 1) supplanting contrast-enhanced CT or MR myelography; 2) replacing conventional T2-FSE sequences in the imaging of degenerative $\mathrm{C}$-spine disease, similar to a recent study examining lumbar spine $\mathrm{MR}$ imaging ${ }^{20}$; 3 ) assessing traumatic C-spine ligamentous injury and nerve root avulsions; and, 4) generating oblique sagittal MPR images for the evaluation of C-spine neural foramina without the time cost associated with T2-FSE oblique imaging. ${ }^{21}$ However, robust comparative effectiveness studies are needed to further characterize the benefits and limits of this sequence's uses.

\section{CONCLUSIONS}

T2-SPACE may be superior to T2-FSE for evaluation of some, but not all, C-spine anatomic structures and shows less degree of CSF flow artifact. This provides further opportunities for this sequence to replace T2-FSE for certain clinical implications or to avoid contrast-enhanced CT or MR myelography.

\section{REFERENCES}

1. Mugler JP 3rd. Optimized three-dimensional fast-spin-echo MRI. J Magn Reson Imaging 2014;39:745-67 CrossRef Medline

2. Senova S, Hosomi K, Gurruchaga JM, et al. Three-dimensional SPACE fluid-attenuated inversion recovery at $3 \mathrm{~T}$ to improve subthalamic nucleus lead placement for deep brain stimulation in Parkinson's disease: from preclinical to clinical studies. J Neurosurg 2016;125:472-80 CrossRef Medline

3. Ciftci E, Anik Y, Arslan A, et al. Driven equilibrium (drive) MR imaging of the cranial nerves V-VIII: comparison with the T2weighted 3D TSE sequence. Eur J Radiol 2004;51:234-40 CrossRef Medline

4. Czerny C, Trattnig S, Baumgartner WD, et al. [MRI of the regions of the inner ear and cerebellopontine angle using a 3D T2-weighted turbo spin-echo sequence. Comparison with conventional 2D T2weighted turbo spin-echo sequences and T1-weighted spin-echo sequences]. [Article in German] Rofo 1997;167:377-83 CrossRef Medline

5. Blizzard DJ, Haims AH, Lischuk AW, et al. 3D-FSE isotropic MRI of the lumbar spine: novel application of an existing technology. J Spinal Disord Tech 2015;28:152-57 CrossRef Medline

6. Fu MC, Buerba RA, Neway WE 3rd, et al. Three-dimensional isotropic MRI of the cervical spine: a diagnostic comparison with conventional MRI. Clin Spine Surg 2016;29:66-71 CrossRef Medline

7. Lee S, Jee WH, Jung JY, et al. MRI of the lumbar spine: comparison of 3D isotropic turbo spin-echo SPACE sequence versus conventional 2D sequences at 3.0 T. Acta Radiol 2015;56:174-81 CrossRef Medline

8. Meindl T, Wirth S, Weckbach S, et al. Magnetic resonance imaging of the cervical spine: comparison of 2D T2-weighted turbo spin echo, 2D T2* weighted gradient-recalled echo and 3D T2-weighted variable flip-angle turbo spin echo sequences. Eur Radiol 2009;19: 713-21 CrossRef Medline

9. Obuchowski NA. Statistical Issues in Study Design. In: Obuchowski NA, Gazelle GS, eds. Handbook for Clinical Trials of Imaging and Image-Guided Interventions. Hoboken: Wiley-Blackwell; 2016:103-25

10. Viera AJ, Garrett JM. Understanding interobserver agreement: the kappa statistic. Fam Med 2005;37:360-63 Medline

AJNR Am J Neuroradiol 38:846-50 Apr 2017 www.ajnr.org 
11. Ross JS. MR imaging of the cervical spine: techniques for two- and three-dimensional imaging. AJR Am J Roentgenol 1992;159:779-86 CrossRef Medline

12. Chokshi FH, Tu RK, Nicola GN, et al. Myelography CPT coding updates: effects of $\mathbf{4}$ new codes and unintended consequences. AJNR Am J Neuroradiol 2016;37:997-99 CrossRef Medline

13. Cowley P. Neuroimaging of spinal canal stenosis. Magn Reson Imaging Clin N Am 2016;24:523-39 CrossRef Medline

14. van Rijn JC, Klemetsö N, Reitsma JB, et al. Observer variation in MRI evaluation of patients suspected of lumbar disk herniation. $A J R$ Am J Roentgenol 2005;184:299-303 CrossRef Medline

15. Stafira JS, Sonnad JR, Yuh WT, et al. Qualitative assessment of cervical spinal stenosis: observer variability on $C T$ and $M R$ images. AJNR Am J Neuroradiol 2003;24:766-69 Medline

16. Braga-Baiak A, Shah A, Pietrobon R, et al. Intra- and inter-observer reliability of MRI examination of intervertebral disc abnormalities in patients with cervical myelopathy. Eur J Radiol 2008;65:91-98 CrossRef Medline
17. Cook C, Braga-Baiak A, Pietrobon R, et al. Observer agreement of spine stenosis on magnetic resonance imaging analysis of patients with cervical spine myelopathy. J Manipulative Physiol Ther 2008;31: 271-76 CrossRef Medline

18. Kuijper B, Beelen A, van der Kallen BF, et al. Interobserver agreement on MRI evaluation of patients with cervical radiculopathy. Clin Radiol 2011;66:25-29 CrossRef Medline

19. Chen W, Wunderlich A, Petrick N, et al. Multireader multicase reader studies with binary agreement data: simulation, analysis, validation, and sizing. J Med Imaging (Bellingham) 2014;1:031011 CrossRef Medline

20. Sayah A, Jay AK, Toaff JS, et al. Effectiveness of a rapid lumbar spine MRI protocol using 3D T2-weighted SPACE imaging versus a standard protocol for evaluation of degenerative changes of the lumbar spine. AJR Am J Roentgenol 2016;207:614-20 CrossRef Medline

21. Freund W, Klessinger S, Mueller M, et al. [Utility of coronal oblique slices in cervical spine MRI: improved detection of the neuroforamina]. [Article in German] Radiologe 2015;55:1000-08 CrossRef Medline 